\title{
Gossamer Superconductivity, New Paradigm?
}

\author{
Hyekyung Won ${ }^{* 1}$, Stephan Haas ${ }^{2}$, Kazumi Maki ${ }^{2,3}$, David Parker ${ }^{2}$, Balazs Dora ${ }^{4}$, and Attila \\ Virosztek $^{4,5}$ \\ ${ }^{1}$ Department of Physics, Hallym University, Chuncheon 200-702, South Korea \\ ${ }^{2}$ Department of Physics and Astronomy, University of Southern California, Los Angeles, CA 90089-0484 \\ USA \\ ${ }^{3}$ Max-Planck Institute for the Physics of Complex Systems, Nöthnitzer Str. 38, D-01187, Dresden, Ger- \\ many \\ ${ }^{4}$ Department of Physics, Budapest University of Technology and Economics, H-1521, Budapest, Hungary \\ ${ }^{5}$ Research Institute for Solid State Physics and Optics, P.O. Box 49, H-1525 Budapest, Hungary
}

\begin{abstract}
Key words cuprate, d-wave superconductivity, d-wave density wave
PACS 74.70.-b

We shall review our recent works on d-wave density wave (dDW) and gossamer superconductivity (i.e. dwave superconductivity in the presence of $\mathrm{dDW}$ ) in high- $\mathrm{T}_{c}$ cuprates and $\mathrm{CeCoIn}_{5}$. a) We show that both the giant Nernst effect and the angle dependent magnetoresistance (ADMR) in the pseudogap phases of the cuprates and $\mathrm{CeCoIn}_{5}$ are manifestations of $\mathrm{dDW}$. b) The phase diagram of high- $\mathrm{T}_{c}$ cuprates is understood in terms of mean field theory, which includes two order parameters $\Delta_{1}$ and $\Delta_{2}$, where one order paremeter is from $\mathrm{dDW}$ and the other from d-wave superconductivity. c) In the optimally to the overdoped region we find the spatially periodic dDW, an analogue of the Fulde-Ferrell-Larkin-Ovchinnikov (FFLO) state, becomes more stable. d) In the underdoped region where $\Delta_{2} / \Delta_{1} \ll 1$ the Uemera relation is obtained within the present model. We speculate that the gossamer superconductivity is at the heart of high- $\mathrm{T}_{c}$ cuprate superconductors, the heavy-fermion superconductor $\mathrm{CeCoIn}_{5}$ and the organic superconductors $\kappa$ $(\mathrm{ET})_{2} \mathrm{Cu}(\mathrm{NCS})_{2}$ and $(\mathrm{TMTSF})_{2} \mathrm{PF}_{6}$.
\end{abstract}

Copyright line will be provided by the publisher

\section{Introduction}

Question: What does strong correlation mean?

Answer: First of all it means the Coulomb dominance; the Coulomb interaction is stronger than that due to phonon exchange. For superconductors this means unconventional order parameters: d-wave,f-wave, g-wave superconductors.

Since the discovery of high- $\mathrm{T}_{c}$ cuprates $\mathrm{La}_{2-x} \mathrm{Ba}_{x} \mathrm{CuO}_{4}$ by Bednorz and Müller [1] in 1986, it appears that the debate over the nature and mechanism of this unusual superconductivity continues. However, d-wave superconductivity as in BCS theory and arising due to anti-paramagnon exchange has been established, at least in the vicinity of the optimal doping.[2] 3] Also from the low temperature thermal conductivity May Chiao et al deduced $\Delta / E_{F}=1 / 10$ and $1 / 14$ for Bi-2212 and YBCO respectively [4] 5]. From these we obtain $\Delta=500 \mathrm{~K}$ and $280 \mathrm{~K}$ for $\mathrm{Bi}-2212$ and $\mathrm{YBCO}$ respectively and $\mathrm{E}_{F} \simeq 5000 \mathrm{~K}$, which is almost universal. Here $\Delta$ is the maximal gap of d-wave superconductivity at $T=0$. Also recently the universality of the Fermi velocity $v=2.3 \times 10^{7} \mathrm{~cm} / \mathrm{sec}$ has been established by the angle resolved photoemission spectrum [6].

From these we conclude that the cuprate superconductors are in the BCS limit, far away from the Bose-Einstein condensation limit, and that the superconducting fluctuation effect should be at most $10 \%$.

* Corresponding author: e-mail: hkwon@hallym.ac.kr 
Therefore theories based on the large superconducting fluctuations [7] 8] appear to be unrealistic. Also in many numerical computations on the cuprates, it was assumed that $\Delta \sim E_{F}$. It is clear that such approximations lead to rather unrealistic predictions. On the contrary, with $\Delta=0.1 E_{F}$ Kato et al recently found hundreds of quasiparticle bound states around a vortex of f-wave superconductors, a model system for $\mathrm{Sr}_{2} \mathrm{RuO}_{4}$ [9]. These bound states are the analogues of Caroli, de Gennes and Matricon bound states around a vortex in s-wave superconductivity [10 [1]]. More recently the STM data around a vortex in $\mathrm{Sr}_{2} \mathrm{RuO}_{4}$ has been reported by Lupien et al [12]. Indeed the observed quasiparticle spectrum is very consistent with the theoretical analysis in [9]. Of course in $\mathrm{Sr}_{2} \mathrm{RuO}_{4} \Delta$ should be less than $0.01 \mathrm{E}_{F}$.

In 1993 Volovik [13] showed that the quasiparticle density of states in the vortex state in d-wave superconductors is calculable within a quasiclassical approximation. This work has been extended into several directions: a) thermodynamic functions; b) thermal conductivity; c) scaling relations; d) for arbitrary field orientation; and e) for a variety of gap functions $\Delta(\mathbf{k})^{\prime} s$ [14, 15, 16, 17, 18, 19. As is well known the gap symmetry and the gap function has been the central issue since the discovery of the heavy-fermion superconductors [20]. Since 2001 Izawa et al have succeeded in determining the superconducting gap functions $\Delta(\mathbf{k})^{\prime} s$ in $\mathrm{Sr}_{2} \mathrm{RuO}_{4}$ [21], $\mathrm{CeCoIn}_{5}$ [22], $\kappa$-(ET) $)_{2} \mathrm{Cu}(\mathrm{NCS})_{2}$ [23], $\mathrm{YNi}_{2} \mathrm{~B}_{2} \mathrm{C}$ [24], $\mathrm{PrOs}_{4} \mathrm{Sb}_{12}$ [25, 26], $\mathrm{UPd}_{2} \mathrm{Al}_{3}$ [27, 28] and $\mathrm{CePt}_{3} \mathrm{Si}$ [29, 30] through the angle-dependent thermal conductivity. For a review of these aspects see [31].

The phase diagrams of high- $\mathrm{T}_{c}$ cuprates and $\mathrm{CeCoIn}_{5}$ are shown in Fig. 1a) and Fig. 1b) respectively. As you may recognize, we have replaced the pseudogap phase with d-wave density wave, which is the
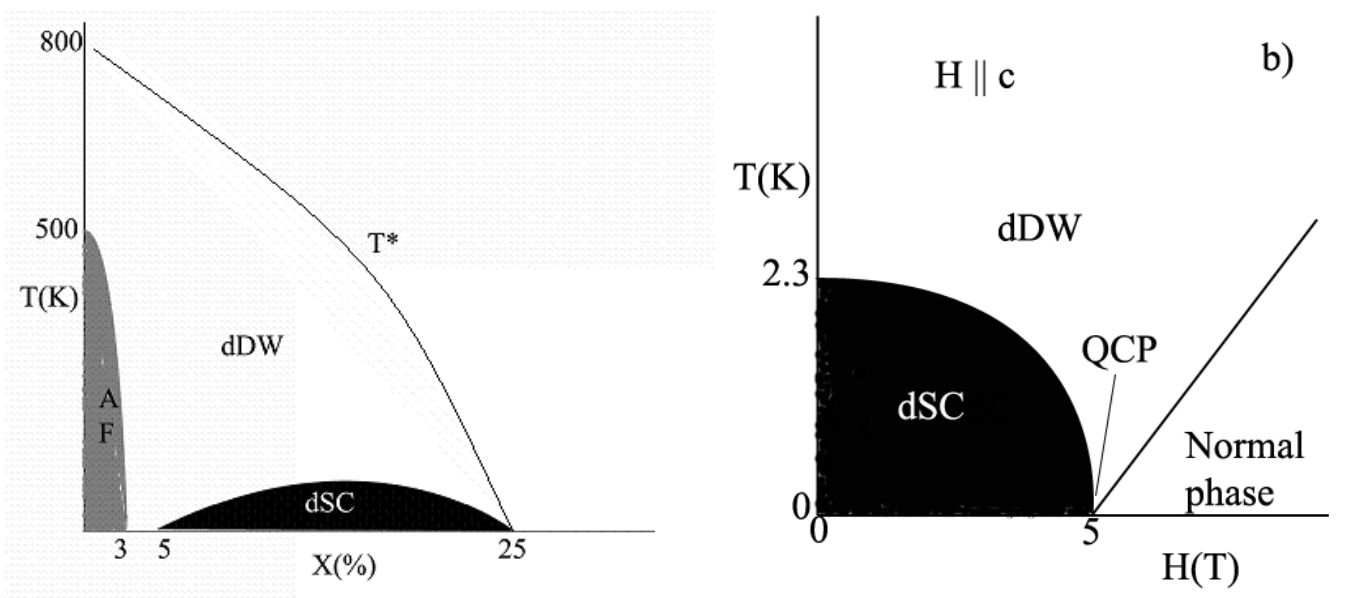

Fig. 1 The phase diagrams for high- $\mathrm{T}_{c}$ cuprates (left) and $\mathrm{CeCoIn}{ }_{5}$

main topic in section 2. Also we have chosen $\mathrm{T}^{*}$ to vanish at the same point where the superconductivity vanishes, suggesting the system has a quantum critical point at $x=25 \%$ [32]. D-wave density wave (dDW) for the pseudogap phase in high- $\mathrm{T}_{c}$ cuprates has been proposed by several people. [33, 34, 35, 36]. However, unlike these authors we do not consider the commensurate $\mathrm{dDW}$ with $\mathrm{Z}_{2}$ - symmetry, which is a descendant of the flux phase [37]. Rather we limit ourselves to the incommensurate dDW with the U(1) gauge symmetry as in the conventional charge density wave [38, 39].

We shall see later that the incommensurate dDW is crucial to understanding the phase diagram in Fig. 1a). Also we shall discuss the recent angle dependent magnetoresistance (ADMR) data in $\mathrm{Y}_{0.68} \mathrm{Pr}_{0.32} \mathrm{CuO}_{4}$ 40,41 and $\mathrm{CeCoIn}_{5}$ [42, which provides strong support for $\mathrm{dDW}$ in these systems. Once one accepts the phase diagrams in Fig. 1, the d-wave superconductivity in the high- $\mathrm{T}_{c}$ cuprates and $\mathrm{CeCoIn}_{5}$ should arise in the presence of dDW. Borrowing the beautiful word from R.B. Laughlin [43] we call these superconductors "gossamer superconductors". Therefore the exploration of the gossamer superconductivity appears to be 
the most urgent [44]. We shall interpret the Uemura relation in the vicinity of $x=5 \%$ in terms of this gossamer superconductivity.

\section{D-wave density waves}

There are many parallels between the cuprates, the heavy-fermion superconductor $\mathrm{CeCoIn}_{5}$ and the organic conductor $\kappa$-(ET) $)_{2} \mathrm{Cu}(\mathrm{NCS})_{2}$ : the quasi-two dimensional Fermi surface, the proximity of the antiferromagnetic phase and d-wave superconductivity [22 45]. In addition d-wave density wave in the pseudogap phase appears to be an additional common feature [41, 42, 46, 47]. In the absence of a magnetic field the Nambu Green function for dDW is given by [41]

$$
G^{-1}(\omega, \mathbf{k})=\omega-\xi(\mathbf{k}) \rho_{3}-\eta(\mathbf{k})-\Delta(\mathbf{k}) \rho_{1}
$$

where the $\rho_{i}$ 's are the Pauli matrices operating on the spinor space. For d-wave charge density wave we can take either $\Delta(\mathbf{k})=\Delta \cos (2 \phi)$ or $\sin (2 \phi)$ with $\tan \phi=k_{y} / k_{x}$ and $\eta(\mathbf{k})=\mu$, the chemical potential, which acts as the imperfect nesting. Further

$$
\xi(\mathbf{k})=v\left(k_{\|}-k_{F}\right)+\frac{v^{\prime}}{c} \cos \left(c k_{z}\right)
$$

where $k_{\|}$is the radial component in the $\mathrm{x}-\mathrm{y}$ plane and $\mathrm{v}$ and $\mathrm{v}^{\prime}$ are the Fermi velocities.

Then the quasiparticle density of states is given by

$$
N(E) / N_{0}=G(x-y)
$$

where

$$
\begin{aligned}
G(x) & =\frac{2 x}{\pi} K(x) \text { for } \mathrm{x} \leq 1 \\
& =\frac{2}{\pi} K\left(x^{-1}\right) \text { for } \mathrm{x}>1 .
\end{aligned}
$$

and $x=E / \Delta, y=\mu / \Delta$ and $\mathrm{K}(\mathrm{x})$ is the complete elliptic integral. $N(E) / N_{0}$ is shown in Fig. 2.

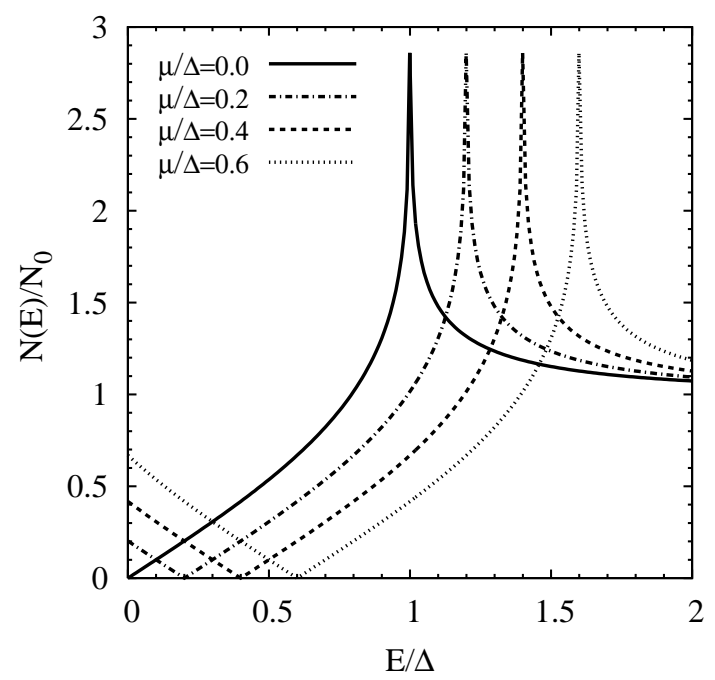

Fig. 2 The quasiparticle density of states for a dDW superconductor 
Note that $N(0) / N_{0} \simeq|\mu| / \Delta$ for $\mu<\Delta$. Therefore the chemical potential provides nonvanishing quasiparticle density of states at $\mathrm{E}=0$. This gives rise to pockets on the Fermi surface at $(\pi, \pi)$ direction in the high- $\mathrm{T}_{c}$ cuprates [48]. Also as we shall see later $\Delta_{2} \leq \mu$ is crucial for the presence of d-wave superconductivity in the middle of dDW. Here $\Delta_{2}$ is the maximum energy gap of a d-wave superconductor.

\section{Landau Quantization}

As noted by Nersesyan et al [49 [50] the quasiparticle spectrum is quantized in the presence of a magnetic field. Let us consider a magnetic field $\mathbf{B}$ applied within the $x^{\prime}-z$ plane tilted by an angle $\theta$ from the $\mathbf{z}$ axis. Also $\hat{x}^{\prime}$ is defined by $\hat{x}^{\prime}=\hat{x} \cos \phi+\hat{y} \sin \phi$. Then the magnetic field is introduced by $\mathbf{k} \rightarrow \mathbf{k}+e \mathbf{A}$ with

$$
\mathbf{A}=B(y \cos \phi-x \sin \phi)(\hat{z} \sin \theta+(\hat{x} \cos \phi+\hat{y} \sin \phi) \cos \theta)
$$

Then for $\mathrm{d}_{x y}$-wave DW, the quasiparticle energies are given by

$$
\begin{aligned}
& E_{1 n}^{ \pm}= \pm \sqrt{2 n e B v_{2}\left|\left(v \cos \theta \cos \phi-v^{\prime} \sin \theta\right) \cos \phi\right|}-\mu \\
& E_{2 n}^{ \pm}= \pm \sqrt{2 n e B v_{2}\left|\left(v \cos \theta \cos \phi+v^{\prime} \sin \theta\right) \cos \phi\right|}-\mu \\
& E_{3 n}^{ \pm}= \pm \sqrt{2 n e B v_{2}\left|\left(v \cos \theta \sin \phi-v^{\prime} \sin \theta\right) \sin \phi\right|}-\mu \\
& E_{4 n}^{ \pm}= \pm \sqrt{2 n e B v_{2}\left|\left(v \cos \theta \sin \phi+v^{\prime} \sin \theta\right) \sin \phi\right|}-\mu
\end{aligned}
$$

Here $\mathrm{n}=0,1,2$,etc. Except for the $\mathrm{n}=0$ Landau level they are doubly degenerate. Also unlike the quasi-one dimensional systems [39], there are 4 branches of the Landau levels [41]. As shown elsewhere these Landau spectra are most readily seen by angle dependent magnetoresistance (ADMR), the nonlinear Hall conductivity and the giant Nernst effect [39 51]. Indeed ADMR appears to provide the most sensitive test of unconventional density wave (UDW) as seen in $\alpha-(\mathrm{ET})_{2} \mathrm{KHg}(\mathrm{SCN})_{4}$ and the Bechgaard salts (TMTSF) ${ }_{2} \mathrm{X}$ with $\mathrm{X}=\mathrm{PF}_{6}$ and $\mathrm{ReO}_{4}$ [52 [53, 54]. Here we present such an analysis of ADMR data provided by $\mathrm{C}$. Almasan, T. Hu and V. Sandu in the pseudogap region in $\mathrm{Y}_{0.68} \mathrm{Pr}_{0.32} \mathrm{CuO}_{4}$ [40 41] and $\mathrm{CeCoIn}_{5}$, which are shown in Fig. 3a and 3b. The electric conductivity is given by [41]

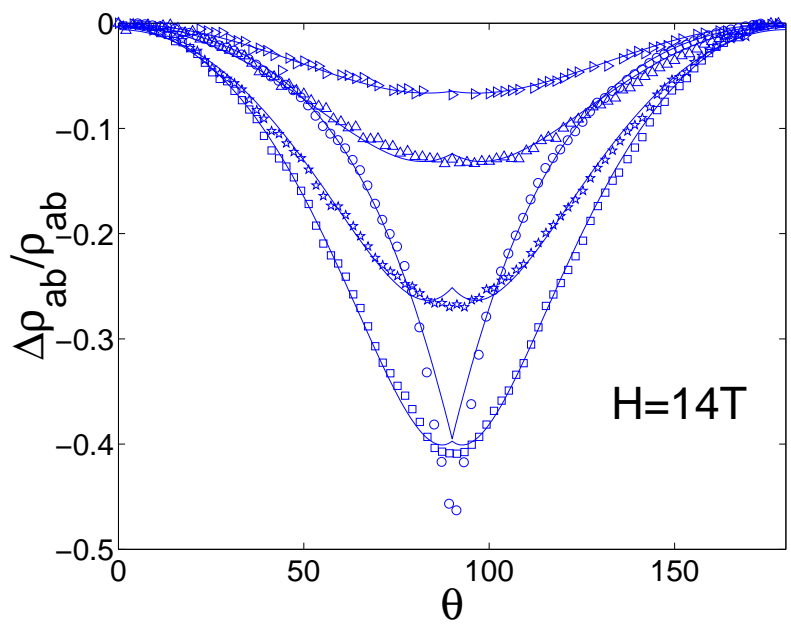

Fig. 3 ADMR of pseudogap region of $\mathrm{Y}_{0.68} \mathrm{Pr}_{0.32} \mathrm{CuO}_{4}$. Curves are for $\mathrm{T}=105 \mathrm{~K}, 75 \mathrm{~K}, 65 \mathrm{~K}, 60 \mathrm{~K}$ and $52 \mathrm{~K}$ from top to bottom. The curve for $\mathrm{T}=52 \mathrm{~K}$ is reduced by a factor of 10 . 


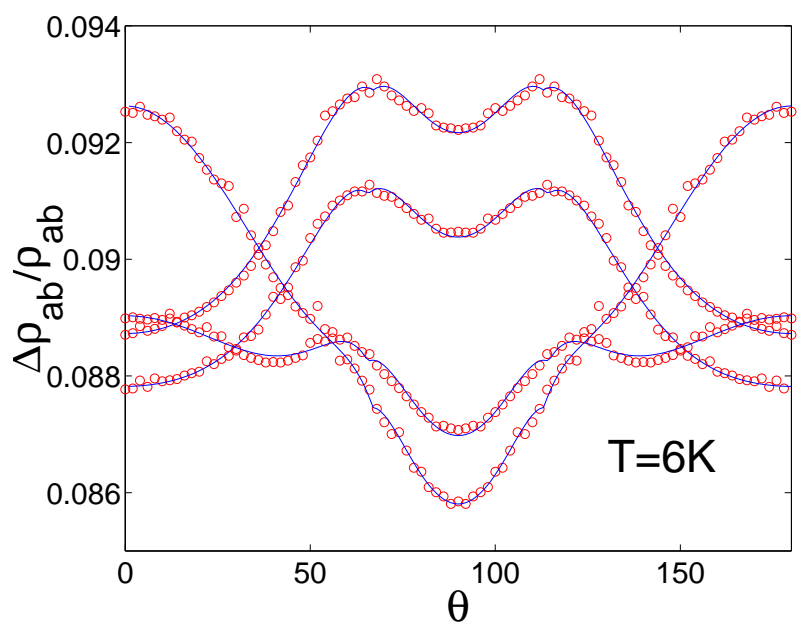

Fig. 4 ADMR of pseudogap region of $\mathrm{CeCoIn}_{5}$, for $\mathrm{H}=3 \mathrm{~T}, 5 \mathrm{~T}, 8 \mathrm{~T}$ and $14 \mathrm{~T}$ from top to bottom [42].

$$
\sigma(B, \theta)=\sum_{n} \sigma_{n} \operatorname{sech}^{2}\left(\beta E_{n} / 2\right)
$$

where $\beta=1 / k_{B} T$ and the sum is over all the Landau lavels. However, when $\beta\left|E_{1}\right| \gg 1$, the two lowest Landau levels suffice. Then we obtain

$$
\sigma(B, \theta)=\sigma_{0}^{\prime}\left(1+\cosh \zeta_{0}\right)^{-1}+\sigma_{1}^{\prime}\left[\frac{1+\cosh x_{1} \cosh \zeta_{0}}{\cosh x_{1}+\cosh \zeta_{0}}+\frac{1+\cosh x_{2} \cosh \zeta_{0}}{\cosh x_{2}+\cosh \zeta_{0}}\right]
$$

where $\zeta_{0}=\beta \mu, x_{1}=\beta \sqrt{2 e B v_{2}\left|v \cos \theta-v^{\prime} \sin \theta\right|}$ and $x_{2}=\beta \sqrt{2 e B v_{2}\left|v \cos \theta+v^{\prime} \sin \theta\right|}$. Here we consider the case $\phi=0$ and $\sigma_{0}^{\prime}$ and $\sigma_{1}^{\prime}$ are $\sigma_{0}$ and $\sigma_{1}$ multiplied by some integer which accounts for the proper degeneracy. From the fitting of Fig. 3a) we obtain $v=2.3 \times 10^{7} \mathrm{~cm} / \mathrm{s}, v^{\prime} / v \leq 0.1, E_{F}=5000 K$, $\Delta=360 K$ and $\mu \simeq 40-60 K$ for $\mathrm{Y}_{0.68} \operatorname{Pr}_{0.32} \mathrm{CuO}_{4}$ with $\mathrm{T}_{c}=55 \mathrm{~K}$. Similarly the data from $\mathrm{CeCoIn}_{5}$ is analyzed in [42]. We find $v=3.3 \times 10^{6} \mathrm{~cm} / \mathrm{s}, v^{\prime} / v \simeq 0.5, E_{F}=500 \mathrm{~K}, \Delta=45 \mathrm{~K}$ and $\mu=8.4 \mathrm{~K}$ for $\mathrm{CeCoIn}_{5}$. These values are consistent with other observations in $\mathrm{CeCoIn}_{5}$ [55]. The Hall conductivity is given similarly by

$$
\sigma_{x y}=-\frac{2 e^{2} \cos ^{2} \theta}{\pi} n(B, T)
$$

with

$$
n(B, T)=\tanh \left(\zeta_{0} / 2\right)+\frac{\sinh \left(\zeta_{0}\right)}{\cosh x_{1}+\cosh \zeta_{0}}+\frac{\sinh \left(\zeta_{0}\right)}{\cosh x_{2}+\cosh \zeta_{0}}+\ldots
$$

A similar expression has been obtained in [54]. The giant Nernst effect in the pseudogap phase of the high$\mathrm{T}_{c}$ cuprates and $\mathrm{CeCoIn}_{5}$ has already been discussed in [46, 47 56]. In conclusion, ADMR, the non-linear Hall conductivity and the giant Nernst effect should provide a clear signature of UDW. 


\section{Gossamer Superconductivity}

Let us consider a simplest coupled equation for $\Delta_{1}(\mathrm{dDW})$ and $\Delta_{2}$ (d-wave superconductivity) [44]:

$$
\begin{aligned}
& \lambda_{1}^{-1}=4 \pi T \sum_{n} \operatorname{Re}\left\langle\frac{f^{2}}{\left[\left(\sqrt{\omega_{n}^{2}+\Delta_{2}^{2} f^{2}}-i \mu\right)^{2}+\Delta_{1}^{2} f^{2}\right]^{1 / 2}}\right\rangle \\
& \lambda_{2}^{-1}=4 \pi T \sum_{n} \operatorname{Re}\left\langle\frac{f^{2}\left(1-\frac{i \mu}{\sqrt{\omega_{n}^{2}+\Delta_{2}^{2} f^{2}}}\right)}{\left[\left(\sqrt{\omega_{n}^{2}+\Delta_{2}^{2} f^{2}}-i \mu\right)^{2}+\Delta_{1}^{2} f^{2}\right]^{1 / 2}}\right\rangle
\end{aligned}
$$

where $\lambda_{1}$ and $\lambda_{2}$ are dimensionless coupling constants, $\mathrm{f}=\cos (2 \phi)$ and $\langle\ldots\rangle$ means $\int_{0}^{2 \pi} \frac{d \phi}{2 \pi}$. A similar set of equations is considered in [36 57]. First let us consider Eq. (14) for $\Delta_{2}=0$. Then we discover that the equation is the same as for a d-wave superconductor in the presence of the Pauli term [58]. Now if one puts $\Delta_{1}=0$, we obtain the equation for $T_{c 1}$ for $\mathrm{dDW}\left(=T^{*}\right)$ as

$$
\ln \left(\frac{T_{c 1}}{T_{c 10}}\right)=\operatorname{Re} \Psi\left(\frac{1}{2}-\frac{i \mu}{2 \pi T_{c 1}}\right)-\Psi\left(\frac{1}{2}\right)
$$

This is shown in Fig. 5. Here $\Psi(z)$ is the digamma function and Eq.(17) is the same as in s-wave super-

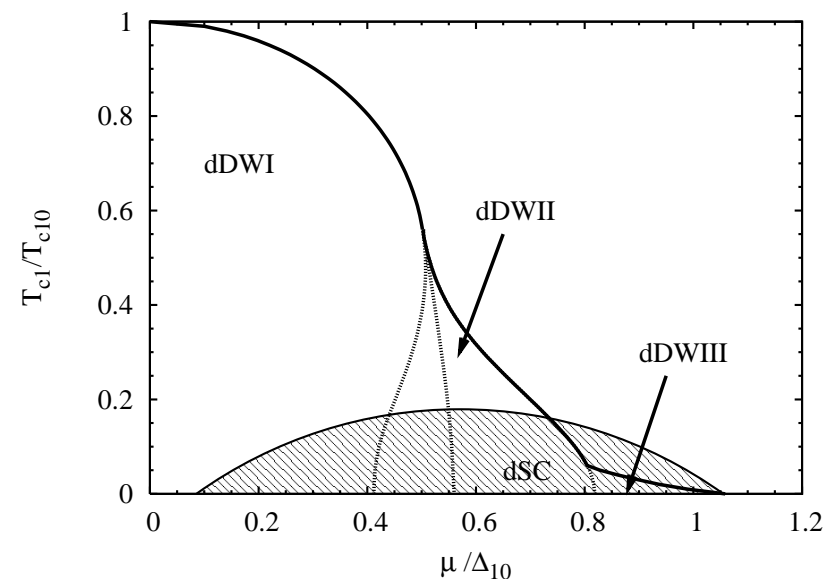

Fig. 5 Proposed phase diagram for high- $\mathrm{T}_{c}$ cuprates

conductors [59, 60]. The figure for $\mathrm{T}_{c 1}$ bends back and there will be no solution for $\mu / \Delta_{10}>0.57$. On the other hand in the region $0.41<\mu / \Delta_{10}<0.57 \mathrm{~T}_{c 1}$ is double-valued. [58]. A similar phase boundary is obtained numerically in [36]. However, the phase boundary for $\mathrm{dDW}$ is extended if we allow the spatial variation of $\Delta: \Delta(\mathbf{r}) \propto \cos (\mathbf{q} \cdot \mathbf{r})$.

This is similar to the Fulde-Ferrell-Larkin-Ovchinnikov (FFLO) state in d-wave superconductors [61], with $\mathrm{T}_{c 1}$ given by

$$
-\ln \left(\frac{T_{c 1}}{T_{c 10}}\right)=\operatorname{Re}\left\langle(1 \pm \cos (2 \phi)) \Psi\left(\frac{1}{2}-\frac{i \mu(1-p \cos \phi)}{2 \pi T_{c 1}}\right)\right\rangle-\Psi\left(\frac{1}{2}\right)
$$

where $\mathbf{p}$ is the new adjustable parameter. Then \pm in Eq. (18) corresponds to $\mathbf{q} \|[100]$ and $\mathbf{q} \|[110]$ and $p=\frac{v|q|}{2 \mu}$. The extended solution is shown in Fig. 5 as well. Then it is more appropriate to split dDW in three separate regions as indicated in Fig. 5. Here we took $\Delta_{10}=1700 \mathrm{~K}$ and $\mathrm{T}_{c 1}=800 \mathrm{~K}$ in accordance with Ref.[62]. Finally we indicate the d-wave superconducting region by a shaded area, which should follow from the set of equations (15) and (16). Also it is possible that dDW III may be submerged under the d-wave superconductivity. 
Since $T_{c 1}>T_{c 2}$ in general, it is natural to assume $\lambda_{1} \geq \lambda_{2}$. Then in the vicinity of $x \simeq 0.5 \%$, where d-wave superconductivity begins to appear, we can assume $\Delta_{2} / \Delta_{1} \ll 1$. Note that $\mu \sim 2300(x-0.0675)$ $\mathrm{K}[63]$ in the whole region. Then combining Eqs. (15) and (16) we find

$$
\begin{aligned}
\lambda_{2}^{-1}-\lambda_{1}^{-1} & \simeq 4 \pi T \mu^{2} \sum_{n}\left\langle\frac{f^{2}}{\left[\omega_{n}^{2}+\Delta^{2} f^{2}\right]^{\frac{3}{2}}}\right\rangle \\
& \simeq \frac{2 \mu^{2}}{\Delta^{2}(T)}\left(1-2(\ln 2) \frac{T}{\Delta_{0}}\right)
\end{aligned}
$$

where $\Delta^{2}(T)=\Delta_{1}^{2}(T)+\Delta_{2}^{2}(T)$. Then Eq.(20) is solved as

$$
\left(\frac{\Delta_{2}(T)}{\Delta_{2}(0)}\right)^{2} \simeq 1-2(\ln 2) \frac{T}{\Delta(0)}\left(\frac{\Delta_{1}(0)}{\Delta_{2}(0)}\right)^{2}
$$

or

$$
T_{c 2} \simeq \frac{1}{2(\ln 2)} \frac{\Delta_{2}^{2}(0)}{\Delta(0)}
$$

On the other hand the superfluid density in the gossamer superconductivity is given by [44]

$$
\rho_{s}(T)=2 \pi T \Delta_{2}^{2}(T) \sum_{n} \operatorname{Re}\left\langle\frac{f^{2}}{\left[\left(\sqrt{\omega_{n}^{2}+\Delta_{2}^{2} f^{2}}-i \mu\right)^{2}+\Delta_{1}^{2} f^{2}\right]^{\frac{3}{2}}}\right\rangle
$$

which gives

$$
\rho_{s}(0) \simeq \frac{\Delta_{2}^{2}(0)}{\Delta^{2}(0)}
$$

Finally we find

$$
T_{c 2}=\frac{1}{2(\ln 2)} \Delta(0) \rho_{s}(0)
$$

Since $\lambda^{-2}(0)=\frac{4 \pi e^{2}}{m^{*}} n \rho_{s}(0)$ the above relation can be interpreted as the celebrated Uemura relation [64], which could not be obtained within the framework of the BCS theory.

Also the present phase diagram suggests that the optimally doped superconductor sits at the boundary of dDW I and dDW II. Of course the present analysis requires further elaboration. Nevertheless, the present model appears to describe qualitatively the phase diagram of high- $\mathrm{T}_{c}$ cuprate superconductors. Also, Fig. 5 suggests naturally that both $\mathrm{dDW}$ III and d-wave superconductivity terminate at $\mu / \Delta_{01}=1.06$ (or x $=25 \%$ ), implying the quantum critical point $(\mathrm{QCP})$ at $\mathrm{x}=25 \%$. We have mentioned previously that the d-wave superconductivity in CeCoIn 5 is also most likely "gossamer".

\section{Conclusions}

We have seen previously that most of the metallic ground states in high- $\mathrm{T}_{c}$ cuprates, heavy-fermion conductors and organic conductors belong to one of the mean field ground states: a) unconventional superconductivity, b) unconventional density wave; or c) the coexistence of both unconventional superconductivity and UDW. The present study suggests that perhaps a) most of the pseudogap phase or "non-Fermi liquid" behaviors are related to UDW; and b) the superconducting phases in both high- $\mathrm{T}_{c}$ cuprates and $\mathrm{CeCoIn}_{5}$ are gossamer; and c) the superconductivity in $\kappa-(\mathrm{ET})_{2}$ salts and in Bechgaard salts (TMTSF) ${ }_{2} \mathrm{PF}_{6}$ also appear to be gossamer [65 66]. In the last system the superconductivity is expected to be triplet and should contain an unconventional spin density wave (USDW) [53 [54]. This suggests that there are a variety of gossamer superconductors, which await our exploration. 
Acknowledgements First of all we thank Carmen Almasan, Tao Hu and Viorel Sandu for providing us the data we used in Fig. 3 and Fig. 4. During the course of this work we have benefitted from enlightening discussions with Carlo di Castro, Andreas Greco, Peter Horsch, Walter Metzner, Alejandro Muramatsu, Maurice Rice, Manfred Sigrist, Peter Thalmeier and Roland Zeyher. HW acknowledges support from the Korean Research Foundation (KRF) through Grant No. R05-2004-000-10814.

\section{References}

[1] J.G. Bednorz and K.A. Müller, Z. Phys. B 64, 180 (1986).

[2] H. Won, S. Haas, D. Parker, and K. Maki, Phys. Stat. Sol. (b) 242, 363 (2005).

[3] H. Won, S. Haas, D. Parker, S. Telang, A. Vànyolos and K. Maki, Proceeding of training school IX at Vietri Sul Mare, also cond-mat/0501463

[4] May Chiao, R.W. Hill, C. Lupien, B. Popic, R. Gagnon and L. Taillefer, Phys. Rev. Lett. 82, 2943 (1999).

[5] May Chiao, Ph.D thesis, McGill University (1999).

[6] X.J. Zhou et al, Nature 423, 398 (2003).

[7] J. Orenstein and A.J. Millis, Science 288, 468 (2000).

[8] S. Kivelson et al, Rev. Mod. Phys. 75, 1201 (2003).

[9] M. Kato, H. Suematsu and K. Maki, Physica C 408-410, 535 (2004).

[10] C. Caroli, P.G. de Gennes and J. Matricon, Phys. Lett. 9, 307 (1964).

[11] C. Caroli and J. Matricon, Physik Kondensierten Materie 3, 380 (1965).

[12] C. Lupien, S.K. Dutta, B.I. Barker, Y. Maeno and J.C. Davis, cond-mat/0503317

[13] G.E. Volovik, JETP Lett. 58, 496 (1993).

[14] C. Kübert and J.P. Hirschfeld, Solid State Comm. 105, 459 (1998).

[15] C. Kübert and J.P. Hirschfeld, Phys. Rev. Lett. 80, 4963 (1998).

[16] I. Vehkter, J.P. Carbotte, E.J. Nicol, Phys. Rev. B 59, 7123 (1999).

[17] H. Won and K. Maki, cond-mat/0004105

[18] T. Dahm, K. Maki and H. Won, cond-mat/0006301

[19] H. Won, S. Haas, D. Parker and K. Maki, cond-mat/0503350

[20] M. Sigrist and K. Ueda, Rev. Mod. Phys. 63, 289 (1991).

[21] K. Izawa, H. Takahashi, H. Yamaguchi, Yuji Matsuda, M. Suzuki, T. Sasaki, T. Fukase, Y. Yoshida, R. Settai and Y. Onuki, Phys. Rev. Lett. 86, 2653 (2001).

[22] K. Izawa, H. Yamaguchi, Yuji Matsuda, H. Shishido, R. Settai and Y. Onuki, Phys. Rev. Lett. 87, 57002 (2001).

[23] K. Izawa, H. Yamaguchi, T. Sasaki and Yuji Matsuda, Phys. Rev. Lett. 88, 027002 (2002).

[24] K. Izawa, K. Kamata, Y. Nakajima, Y. Matsuda, T. Watanabe, M. Nohara, H. Takagi, P. Thalmeier and K. Maki, Phys. Rev. Lett. 89, 137006 (2002).

[25] K. Izawa, Y. Nakajima, J. Goryo, Y. Matsuda, S. Osaki, H. Sugawara, H. Sato, P. Thalmeier and K. Maki, Phys. Rev. Lett. 90, 117001 (2003).

[26] K. Maki, S. Haas, D. Parker, H. Won, K. Izawa and Y. Matsuda, Europhys. Lett. 65, 720 (2004).

[27] T. Watanabe et al, Phys. Rev. B 70, 184502 (2004).

[28] H. Won, D. Parker, K. Maki, T. Watanabe, K. Izawa and Y. Matsuda, Phys. Rev. B 70, 140509 (2004).

[29] K. Izawa, Y. Kasahara, Y. Matsuda, K. Behnia, T. Yasuda, R. Settai and Y. Onuki, Phys. Rev. Lett. 94, 197002 (2005).

[30] K. Maki and H. Won, preprint.

[31] K. Maki, S. Haas, D. Parker and H. Won, Chinese J. Phys. 43, 532 (2005).

[32] M. Sutherland et al, Phys. Rev. B 67, 174520 (2003).

[33] E. Capelutti and R. Zeyher, Phys. Rev. B 59, 6475 (1999).

[34] L. Benfatto, S. Caprara and C. Di Castro, Eur. Phys. J. B 17, 95 (2000).

[35] S. Chakravarty, R.B Laughlin, D.K. Morr and C. Nayak, Phys. Rev B 63, 094503 (2001).

[36] R. Zeyher and A. Greco, Phys. Stat. Sol. (b) 242, 356 (2005).

[37] I. Affleck and J.B. Marston, Phys. Rev. B 37, 3774 (1988).

[38] G. Grüner, "Density Waves in Solids" (Addison-Wesley, Reading 1994).

[39] B. Dora, K. Maki and A. Virosztek, Mod. Phys. Lett. B 18, 327 (2004).

[40] V. Sandu, E. Cimpoiasu, T. Katuwai, Shi Li, M.B. Maple and C.C. Almasan, Phys. Rev. Lett. 93, 177005 (2004).

[41] B. Dora, K. Maki, A Virosztek, preprint.

[42] T. Hu, H. Xiao, V. Sandu, C.C. Almason, K. Maki, B. Dora, T.A. Sayles and M.B. Maple, preprint.

[43] R.B. Laughlin, cond-mat/0209269 
[44] S. Haas, K. Maki, T. Dahm and P. Thalmeier, Curr. Appl. Phys. (in press).

[45] H. Aoki et al, J. Phys. Cond. Matt, 16, L13 (2004).

[46] R. Bel et al, Phys. Rev. Lett 92, 217002 (2004).

[47] B. Dora, K. Maki, A. Vànyolos and A. Virosztek, Phys. Stat. Sol. (b) 242, 404 (2005); Phys. Rev. B 71, 172502 (2005).

[48] J.C. Campuzano, H. Ding, M.R. Norman, M. Randeira, Physica B 259-261, 517 (1999).

[49] A.A. Nersesyan, G.I. Vachnadze, J. Low Temp. Phys. 77, 293 (1989).

[50] A.A. Nersesyan, G.I. Japaridze and I.G. Kimeridze, J. Phys. Cond. Matt. 3, 3353 (1991).

[51] M. Basletić, B. Korin-Hamzić, A. Hamzić and K. Maki, Synth. Metals 141, 99 (2004).

[52] K. Maki et al, Phys. Rev. Lett. 90, 256402 (2003).

[53] W. Kang, H.Y. Kang, Y.J. Jo and S. Uji, Synth. Metals 133-134, 13 (2003).

[54] B. Dora, K. Maki, A. Vànyolos and A. Virosztek, Europhys. Lett. 67, 1024 (2004).

[55] H. Won, K. Maki, S. Haas, N. Oeschler, F. Weickert and P. Gegenwart, Phys. Rev B 69, 180504(R) (2004).

[56] K. Maki, B. Dora, A. Vànyolos and A. Virosztek, Curr. Appl. Phys. 4, 693 (2004).

[57] P. Thalmeier and G. Zwicknagl, in Handbook on the Physics and Chemistry of Rare Earths, edited by K. Gschneider, J.-C. Bünzli, and V. Pecharsky (Elsevier, Amsterdam, 2005), Vol 34, chap. 219.

[58] H. Won, H. Jang and K. Maki, cond-mat/9901252

[59] G. Sarma, J. Phys. Chem. Solids 24, 1629 (1963).

[60] K. Maki in "Superconductivity", Vol. II edited by R.D. Parks (Marcel-Dekker, New York, 1969).

[61] K. Maki and H. Won, Czech J. Phys. 46, 1033 (1996); Physica B 322, 315 (2002).

[62] A.V. Pimenov, A.V. Boris, Li Yu, V. Hinkov, Th. Wolf, J.L. Tallon, B. Keimer and C. Berndhard, Phys. Rev. Lett. 94, 227003 (2005).

[63] H. Won and K. Maki, Physica B 206-207, 664 (1995).

[64] Y.J. Uemura, Physica B 169, 99 (1991).

[65] M. Pinterić, S. Tomić and K. Maki, Physica C 408-410, 75 (2004).

[66] I.J. Lee, S.E. Brown, W. Yu, M.J. Naughton and P. Chaikin, Phys. Rev. Lett. 94, 197001 (2005). 